\title{
Future Insight to Mannuronic Acid (M2000) in Treatment of Neurodegenerative Diseases
}

\author{
Soheil Najafi and Abbas Mirshafiey* \\ Department of Immunology, Tehran University of Medical Sciences, Iran
}

*Corresponding author: Abbas Mirshafiey, Department of Immunology, School of Public Health, Tehran University of Medical Sciences, Tehran, Iran.

Received Date: December 16, 2019

Published Date: January 06, 2020

\begin{abstract}
The $\beta$-D-Mannuronic acid (M2000) as a novel immunosuppressive drug, patented (PCT/EP2017/067920), has immunosuppressive effects. M2000 has shown a notable efficacy in experimental models of multiple sclerosis (MS), rheumatoid arthritis, and nephrotic syndrome. The influence of M2000 on myelin basic protein (MBP) induced experimental autoimmune encephalomyelitis (EAE), an animal model of multiple sclerosis, was evaluated. The results of this study demonstrated that the treatment of EAE with M2000 could significantly decline disease development both prophylactically and therapeutically. The onset and symptoms of EAE in Lewis rats could be suppressed following the administration of M2000. In another study, the effect of M2000 on the treatment of Alzheimer's disease (AD) was performed by Morris water maze experiment, and the immunological evaluations were performed by Western blot, apoptosis (procaspase-3, Bax/Bcl2, P53), enzymatic (superoxide dismutase [SOD]), and nonenzymatic oxidative stress (malondialdehyde [MDA]) tests. Our data showed that M2000 can reduce the amount of Bax/Bcl2, P53, MDA, and SOD, as well as it normalized the level of procaspase 3. Also, in a clinical trial, we assessed the efficacy and safety outcomes in MS treated patients with mannuronic acid compared to the conventional drug. As compared with the conventional drug, mannuronic acid (M2000) improved the risk of disability progression (based on MRI and EDSS assessment). This clinical trial demonstrated the efficacy and safety of mannuronic acid in patients with MS. Our results suggest M2000 is a potential therapeutic agent for the treatment of MS and AD.
\end{abstract}

Keywords: M2000; Mannuronic acid; Multiple sclerosis; Alzheimer's disease; EAE

\section{Mini Review}

Multiple sclerosis (MS), the principal inflammatory demyelinating disease of the central nervous system (CNS), is believed to have an immunopathological etiology arising from gene-environment interactions $[1,2]$ affecting approximately $0.1 \%$ of the population in the northern part of the world. The factors behind the initiation of the inflammatory response are not known at present, but MS is considered a complex disease depending on genetic as well as environmental factors. Experimental autoimmune encephalomyelitis (EAE) is the current experimental rodent model for multiple sclerosis. Disease is induced in genetically susceptible mice or rats by immunization with myelin proteins or peptides, resulting into the infiltration of leukocytes into the CNS [3]. In spite that MS results to severe disability, targeted effective therapies for treatment of this disease is lacking. Even more frustrating is the fact that the basic mechanisms underlying the disease process have not been understood in order to design an appropriate therapeutic tool. The principal problem in treatment of MS is the notable side effects of current therapies. The administration of mitoxantrone [4,5] glatiramer acetate [6-8], interferons (IFNs) [9-11], corticosteroids [12], and other immunosuppressive [13,14] and anti- inflammatory drugs [15-18], in addition to their therapeutic effects, commonly cause diverse side effects. Most common among the side effects of IFN are "flu- like" symptoms. Also, depression is a suspected side effect of therapy with IFN- beta, since IFNs affect neurochemical pathways putatively involved in the etiology of depression. In this study we assess the therapeutic potency of M2000 (b-D-mannuronic acid), as a novel designed nonsteroidal anti-inflammatory drug (NSAID) with immunosuppressive property in an experimental model of multiple sclerosis, multiple sclerosis and alzheimer's disease. The therapeutic potency of M2000 (b-D-mannuronic acid), a novel designed nonsteroidal antiinflammatory drug with immunosuppressive property in T-cellmediated autoimmune disease, was tested [19-23]. The influence of M2000 on myelin basic protein (MBP)-induced experimental 
autoimmune encephalomyelitis (EAE), an animal model of multiple sclerosis, was evaluated [24]. M2000 at two doses, 40 and $80 \mathrm{mg} /$ $\mathrm{kg} /$ day, was administered intraperitoneally (i.p.) to the prevention and treatment groups, respectively. Onset of i.p. injections of M2000 to prophylactic and therapeutic groups was day-1 and day-7 postimmunization. The WEHI-164 cell line was applied for evaluating the tolerability of M2000. The results of this experiment demonstrated that the treatment of EAE with M2000 could significantly suppress disease development both prophylactically and therapeutically; the onset and symptoms of EAE in Lewis rats suppressed following the administration of M2000. Clinical improvement was accompanied by a significant decrease in mean numbers of vessels with perivascular cellular infiltration in M2000-treated rats compared with non-treated control. Disease suppression was associated with a marked suppression of MBPspecific T-cell reactivity in vitro, without any evidence for a generalized impairment of T-cell activity. Furthermore, M2000 demonstrated a very high tolerability compared with certain steroidal and non-steroidal anti-inflammatory drugs. Collectively, our data suggested that M2000 might supply a novel therapeutic option for T-cell-mediated autoimmune diseases in animal models and possibly in humans [24]. Histopathological evaluation of brain and spinal cord sections in treated and non-treated groups showed that the mean number of vessels with perivascular cellular infiltration in brain $(0.57 \pm 0.79)$ and spinal cord $(2.0 \pm 2.65)$ in M2000-treated rats were significantly less than non-treated control with mean values of $(2.43 \pm 3.41)$ for brain and $(10.71 \pm 8.76)$ for spinal cord, respectively [24]. The mechanism of action of M2000 is due in part to inhibition of matrix metalloproteinase activity and/or expression and its apoptotic efficacy [20], which is parallel with immunoprotective mechanism of IFN-beta [10]. Moreover, the lymphoid cells from draining lymph nodes of M2000-treated MBPsensitized rats showed in vitro diminished proliferative response that provides understanding of immunosuppressive property of this drug. Additionally, together with our histological findings, the data suggested that the relevant immunosuppressive mechanisms of M2000 may be suppression of migration of leukocytes into the CNS and to some extent its inhibitory effect on T-cell proliferation. Our findings showed that M2000 (b-D-mannuronic acid) might provide a novel therapeutic option for treatment of multiple sclerosis. And due to its high tolerability, it can be recommended as a safe immunosuppressive agent for long-term administration [24]. In another study, the effect of M2000 on the treatment of Alzheimer's disease (AD) was performed by Morris water maze experiment, and the immunological assessments were carried out by Western blot, apoptosis (procaspase-3, Bax/Bcl, P53), enzymatic (superoxide dismutase [SOD]), and nonenzymatic oxidative stress (malondialdehyde [MDA]) tests [25]. We found that pretreatment of $\mathrm{AD}$ in the rat model by M2000 had a potent efficacy on rat behavior and also it caused a significant inhibition of amyloid plaque production. In addition, our data demonstrated that M2000 can decrease the amount of $\mathrm{Bax} / \mathrm{Bcl}_{2}, \mathrm{P} 53, \mathrm{MDA}$, and SOD, as well as it normalized the level of procaspase-3. Our data suggested that M2000 is a potential therapeutic agent for the treatment of
AD. In our study, rats were divided into 4 groups-normal control group (C), sham operation group (S) in which phosphate-buffered saline (PBS) was injected to the intrahippocampus, Alzheimer's group (Ab) in which $50 \mathrm{ng} / \mathrm{mL}$ per side of $\mathrm{Ab}$ was injected to the intrahippocampus by Hamilton syringe, and M2000 group (Ab plus M) that in addition to Ab injection, they obtained M2000 daily via water utility. The M2000 was given to the rats for 6 weeks (from 2 weeks before $\mathrm{Ab}$ injection to 4 weeks after $\mathrm{Ab}$ injection) [25]. Bax is one of the proteins that have a significant role in the development of apoptosis and antiapoptotic protein such as Bcl2, which has an important role in controlling apoptosis pathway. In our experiment, $\mathrm{Ab}$ injection significantly increased Bax expression $(\mathrm{P}<.0001)$ and decreased Bcl2 expression $(\mathrm{P}<.01)$. Consequently, the Bax/Bcl2 ratio significantly increased compared to the control group $(\mathrm{P}<$ 0001). In the group that received M2000, this ratio declined ( $\mathrm{P}<$ .0001) in comparison with the Ab group and did not demonstrate a significant difference compared to the control group.

Also our data revealed that Ab-induced free radical and oxidative stress had a vital role in the pathogenesis of isere AD. Reactive oxidative species (ROS) cause the lipid and protein oxidation with increasing the level of MDA as a product of lipid peroxidation. In several studies, the level of MDA has been mounted in the hippocampus tissue, plasma, and cerebrospinal fluid in patients with $\mathrm{AD}$ [26]. On the other hand, harmful effects of ROS can be neutralized by antioxidant systems such as SOD, CAT, and glutathione. The SOD and CAT are the first-line items against toxicity of ROS. Various studies have revealed the increased or decreased levels of these enzymes in hippocampus and plasma of patients with AD [27]. The results of our study showed that the levels of both SOD and MDA had increased in the Ab group, indicating the defense mechanism against the oxidative stress Although, the group treated by M2000 showed a remarkable reduction in the level of SOD and MDA, which means that the M2000 has a successful impact on the decline of oxidative stress and free radical side effects.

In this experiment, escape latency had a significant reduction in the $\mathrm{Ab}$ plus $\mathrm{M}$ group, which meant that the intake of M2000 might develop learning procedure in $\mathrm{AD}$. The other variable which measured were travel distance and probe trial in which both had a significant decrease in the Ab plus M group compared to the $\mathrm{Ab}$ group. These results of improvement in behavioral procedure led to this conclusion that M2000 could have a potent influence on the AD treatment [25]. Moreover, in our recent study, in a 6-month, randomized controlled, phase II trial, we enrolled patients who had secondary progressive multiple sclerosis (SPMS), were 21 to 54 years of age, with a score of 1 to 7 on EDSS (higher degrees indicate more disability), and who had two or more relapses in the previous year. Patients received orally two doses of $500 \mathrm{mg}$ daily of $\beta$-D-Mannuronic acid. Endpoints were measured with magnetic resonance imaging (MRI) and Expanded Disability Status Scale and compared to the conventional drug (interferon beta-1a, interferon beta-1b), in a phase 2 trial of multiple sclerosis at the beginning and end of the study. 
A total of 25 (92.5\%) of the M2000 treated patients completed the study. M2000 decreased the disability progression over the 6-month period. M2000 had a better performance compared to the conventional drug regarding to MRI-related measurements. Also, this medicine had no particular side effects.

As compared with the conventional drug, mannuronic acid (M2000) improved the risk of disability progression (based on MRI and EDSS assessment). This clinical trial demonstrated the efficacy and safety of mannuronic acid in patients with SPMS (Registered Clinical Trials number, IRCT2017042313739N6). In conclusion, the oral administration of mannuronic acid (M2000) had better efficacy than conventional drug during this 6-month trial in SPMS patients. MRI evidence in SPMS patients and the number of T2 lesions were declined by oral administration of M2000. Furthermore, EDSS score in M2000-treated group comparing to the conventional drug group was reduced significantly. This study demonstrated that M2000 is safe, beneficial and totally well tolerated in patients with multiple sclerosis disease. Moreover, the occurrence of adverse events during the clinical trial was mild and low in the M2000-treated patients. Hence, the reported side effects of this drug are negligible.

\section{Conflict of Interest Statement}

The authors declare that they have no conflicts of interest.

\section{Acknowledgement}

None.

\section{References}

1. Teuscher C, Bunn JY, Fillmore PD, Butterfield RJ, Zachary JF, et al. (2004) Gender, age, and season at immunization uniquely influence the genetic control of susceptibility to histopathological lesions and clinical signs of experimental allergic encephalomyelitis: implications for the genetics of multiple sclerosis. Am J Pathol 165(5): 1593-1602.

2. Najafi S, Mirshafiey A (2015) The Effect of Activated Microglia in Progression of Multiple Sclerosis. International Trends in Immunity 3(4): 96-104

3. Andersson A, Covacu R, Sunnemark D, Danilov AI, Dal Bianco A, et al. (2008) Pivotal advance: HMGB1 expression in active lesions of human and experimental multiple sclerosis. J Leukoc Biol 84(5): 1248-1255.

4. Edan G, Morrissey S, Le Page E (2004) Rationale for the use of mitoxantrone in multiple sclerosis. J Neurol Sci 223(1): 35-39.

5. Tanasescu R, Debouverie M, Pittion S, Anxionnat R, Vespignani H (2004) Acute myeloid leukaemia induced by mitoxantrone in a multiple sclerosis patient. J Neurol 251(6): 762-763.

6. Soos N, Shakery K, Mrowietz U (2004) Localized panniculitis and subsequent lipoatrophy with subcutaneous glatiramer acetate (Copaxone) injection for the treatment of multiple sclerosis. Am J Clin Dermatol 5(5): 357-359.

7. Simpson D, Noble S, Perry C (2002) Glatiramer acetate: a review of its use in relapsing-remitting multiple sclerosis. CNS Drugs 16(12): 825850.

8. Ziemssen T, Neuhaus O, Farina C, Hartung HP, Hohlfeld R (2002) Treatment of multiple sclerosis with glatiramer acetate. Current aspects of mechanisms of action, pharmacokinetics, adverse effect profile and clinical studies. Nervenarzt 73(4): 321-331.
9. Etheridge LJ, Beverley DW, Ferrie C, McManus E (2004) The use of interferon beta in relapsing-remitting multiple sclerosis. Arch Dis Child 89(8): 789-791.

10. Billiau A, Kieseier BC, Hartung HP (2004) Biologic role of interferon beta in multiple sclerosis. J Neurol 251 Suppl 2: II10-4.

11. Lange-Asschenfeldt C, Boor S, Kahaly GJ, Thomke F (2004) Autoimmune functional disorders of the thyroid during interferon-beta-1b treatment in patients with multiple sclerosis. Case report and literature review. Nervenarzt 75(6): 589-594

12. Pozzilli C, Marinelli F, Romano S, Bagnato F (2004) Corticosteroids treatment. J Neurol Sci 223(1): 47-51.

13. Fernandez O, Fernandez V, De Ramon E (2004) Azathioprine and methotrexate in multiple sclerosis. J Neurol Sci 223(1): 29-34.

14. Jeffery DR (2004) The argument against the use of cyclophosphamide and mitoxantrone in the treatment of multiple sclerosis. J Neurol Sci 223(1): 41-46.

15. Rio J, Montalban X (2000) Ibuprofen treatment versus gradual introduction of interferon beta-1b in patients with MS. Neurology 54(8): 1710.

16. Furlan R, Kurne A, Bergami A, Brambilla E, Maucci R, et al. (2004) A nitric oxide releasing derivative of flurbiprofen inhibits experimental autoimmune encephalomyelitis. J Neuroimmunol 150(1-2): 10-19.

17. Moon C, Ahn M, Jee Y, Heo S, Kim S, Kim H, et al. (2004) Sodium salicylateinduced amelioration of experimental autoimmune encephalomyelitis in Lewis rats is associated with the suppression of inducible nitric oxide synthase and cyclooxygenases. Neurosci Lett 356(2): 123-126.

18. Reder AT, Thapar M, Sapugay AM, Jensen MA (1994) Prostaglandins and inhibitors of arachidonate metabolism suppress experimental allergic encephalomyelitis. J Neuroimmunol 54(1-2): 117-127.

19. Mirshafiey A, Cuzzocrea S, Rehm BH, Matsuo H (2005) M2000: a revolution in pharmacology. Med Sci Monit 11(8): PI53-63.

20. Mirshafiey A, Khorramizadeh MR, Saadat F, Rehm BH (2004) Chemopreventive effect of M2000, a new anti-inflammatory agent. Med Sci Monit 10(10): PI105-9.

21. Mirshafiey A, Rehm BH, Sahmani AA, Naji A, Razavi A (2004) M-2000, as a new anti-inflammatory molecule in treatment of experimental nephrosis. Immunopharmacol Immunotoxicol 26(4): 611-619.

22. Mirshafiey A, Rehm B, Abhari RS, Borzooy Z, Sotoude M, et al. (2007) Production of M2000 (beta-d-mannuronic acid) and its therapeutic effect on experimental nephritis. Environ Toxicol Pharmacol 24(1): 6066.

23. Fattahi MJ, Abdollahi M, Agha Mohammadi A, Rastkari N, Khorasani $\mathrm{R}$, et al. (2015) Preclinical assessment of beta-d-mannuronic acid (M2000) as a non-steroidal anti-inflammatory drug. Immunopharmacol Immunotoxicol 37(6): 535-540.

24. Mirshafiey A, Matsuo H, Nakane S, Rehm BH, Koh CS, et al. (2005) Novel immunosuppressive therapy by M2000 in experimental multiple sclerosis. Immunopharmacol Immunotoxicol 27(2): 255-265.

25. Athari Nik Azm S, Vafa M, Sharifzadeh M, Safa M, Barati A, et al. (2017) Effects of M2000 (D-Mannuronic Acid) on Learning, Memory Retrieval, and Associated Determinants in a Rat Model of Alzheimer's Disease. Am J Alzheimers Dis Other Demen 32(1): 12-21.

26. Mangialasche F, Polidori MC, Monastero R, Ercolani S, Camarda C, et al. (2009) Biomarkers of oxidative and nitrosative damage in Alzheimer's disease and mild cognitive impairment. Ageing Res Rev 8(4): 285-305.

27. Sonnen JA, Breitner JC, Lovell MA, Markesbery WR, Quinn JF, et al. (2008) Free radical-mediated damage to brain in Alzheimer's disease and its transgenic mouse models. Free Radic Biol Med 45(3): 219-230. 